Short communication

\title{
Tricellular adherens junctions provide a cell surface delivery platform for connexin 26/30 oligomers in the cochlea
}

\author{
Jean Defourny*, Marc Thiry \\ GIGA-Neurosciences, Unit of Cell and Tissue Biology, University of Liège, C.H.U B36, B-4000 Liège, Belgium
}

\section{A R T I C L E I I N F O}

\section{Article history:}

Received 6 July 2020

Revised 12 November 2020

Accepted 25 November 2020

Available online 30 November 2020

\section{Keywords:}

Cochlea

Gap junctions

Connexin 26

Connexin 30

Tricellular junctions

$\mathrm{N}$-cadherin

\begin{abstract}
A B S T R A C T
In the cochlea, connexins 26 (Cx26) and 30 (Cx30) largely co-assemble into heteromeric gap junctions, which connect adjacent non-sensory epithelial cells. These channels are believed to ensure the rapid removal of $\mathrm{K}^{+}$away from the base of sensory hair cells, resulting in $\mathrm{K}^{+}$recycling back to the endolymph to maintain cochlear homeostasis. Many of the mutations in GJB2 and GJB6, which encode CX26 and CX30, impair the formation of membrane channels and cause autosomal hearing loss in humans. Although recent advances have been made, several important questions remain about connexin trafficking and gap junction biogenesis. Here we show that tricellular adherens junctions present at the crossroad between adjacent gap junction plaques, provide an unexpected cell surface delivery platform for Cx26/Cx30 oligomers. Using an in situ proximity ligation assay, we detected the presence of nonjunctional Cx26/Cx30 oligomers within lipid raft-enriched tricellular junction sites. In addition, we observed that cadherin homophilic interactions are critically involved in microtubule-mediated trafficking of Cx26/Cx30 oligomers to the cell surface. Overall, our results unveil an unexpected role for tricellular junctions in the trafficking and assembly of membrane channels.
\end{abstract}

(C) 2020 Published by Elsevier B.V.

\section{Introduction}

Hearing loss is the most common congenital sensory deficit. About $1-3$ in 1000 children are affected at birth or during early childhood by severe hearing loss, which is defined as prelingual deafness, with at least half of all cases attributable to genetic causes (Korver et al., 2017). Mutations in GJB2 and GJB6, which encode connexins 26 and 30 (CX26 and CX30) involved in inner ear homeostasis, are found in patients with autosomal dominant or recessive non-syndromic hearing loss (del Castillo et al., 2002; Grifa et al., 1999; Kelsell et al., 1997). Beside these non-syndromic forms of deafness, GJB2 and GJB6 mutations also cause several types of skin disorders which are associated or not with hearing deficits (Xu and Nicholson, 2013). In mammals, sounds are perceived through mechanosensory hair cells located within the sensory epithelium of the cochlea (i.e. the organ of Corti). Within the organ of Corti, sensory inner and outer hair cells and non-sensory supporting cells are organized in a regular mosaic pattern that extends along the basal-to-apical axis of the cochlear duct. Cx26 and Cx30 gap junction proteins are believed to ensure the rapid removal of $\mathrm{K}^{+}$away from the base of sensory hair cells, resulting in the recycling of this ion back to the endolymph to maintain

\footnotetext{
* Corresponding author.

E-mail address: jean.defourny@uliege.be (J. Defourny).
}

cochlear homeostasis (Zdebik et al., 2009). However, gap junctions may serve additional roles in the cochlea. There is increasing evidence that intercellular fluxes of second messengers such as inositol phosphates and $\mathrm{Ca}^{2+}$ ions may regulate cochlear physiology. Indeed, the impaired transfer of the $\mathrm{Ca}^{2+}$-mobilizing molecule inositol 1,4,5-trisphosphate has been suggested as a cause of recessive deafness due to a specific GJB2 mutation (Beltramello et al., 2005). In addition, gap junctions within the cochlear sensory epithelium of immature mice are permeable to fluorescent analogues of D-glucose (Chang et al., 2008), pointing to a role for connexins in the transport of energy substrates. In the cochlea, Cx26 and Cx30 largely co-assemble into heteromeric channels, which form a syncytium extending from the spiral limbus to the cochlear spiral ligament (Ahmad et al., 2003; Sun et al., 2005). Although recent advances have been made towards understanding gap junction assembly mechanisms in the cochlea (Defourny et al., 2019b,c), some important questions remain about connexin trafficking pathways and gap junction biogenesis. Indeed, beside mutations that affect the $\mathrm{Cx} 26 / \mathrm{C} \times 30$ channel function itself, many of the disease-causing mutations in GJB2 or GJB6 impair the trafficking and delivery of connexin oligomers to the cell surface, what prevents the formation of membrane channels (Ambrosi et al., 2013; Berger et al., 2014; Hoang Dinh et al., 2009; Xu and Nicholson, 2013). Thus, deciphering the trafficking pathway of cochlear Cx26/Cx30 oligomers 
A

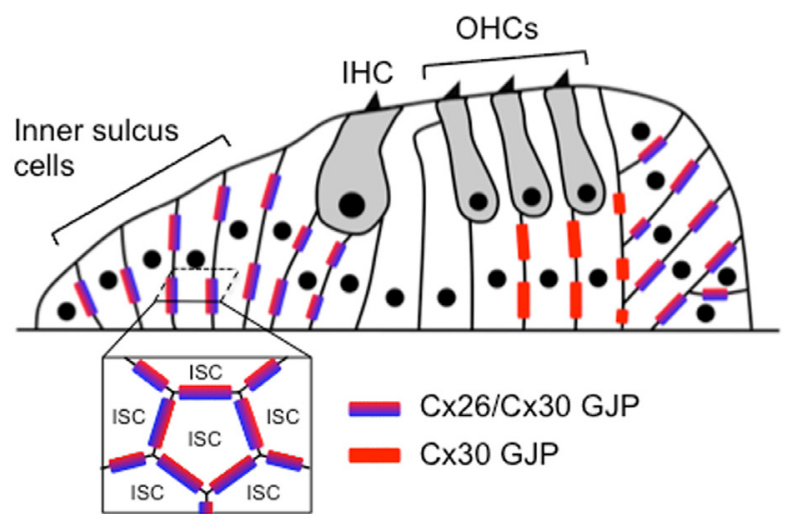

B

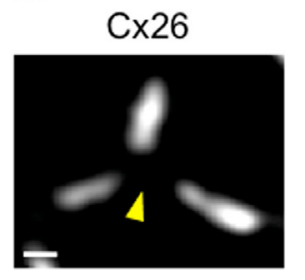

C

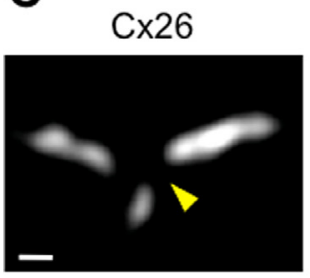

$\mathrm{N}$-cadherin

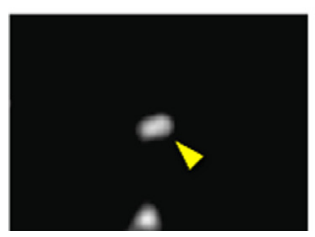

Cholera Toxin B

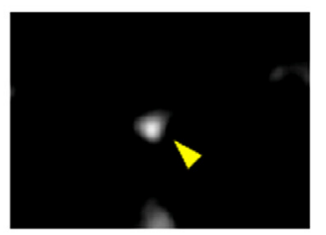

$\mathrm{C} \times 26 /$ $\mathrm{CT} \times \mathrm{B} / \mathrm{N}$-cad.

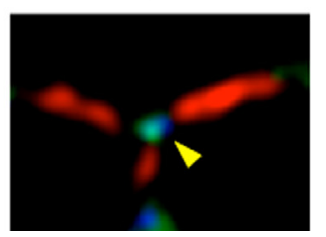

Cholera Toxin B

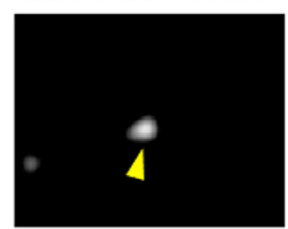

D

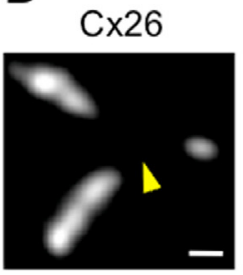

$$
\text { Tubulin }
$$

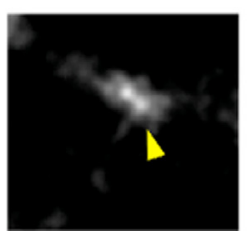

$\mathrm{C} \times 26 /$

$\mathrm{C} \times 30 / \mathrm{CT} \times \mathrm{B}$

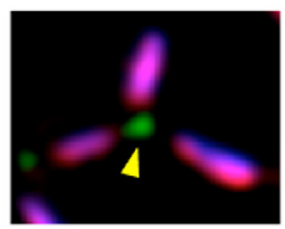

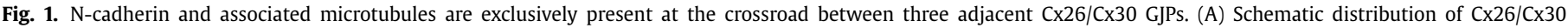

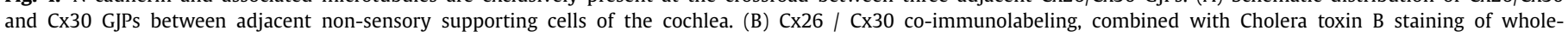

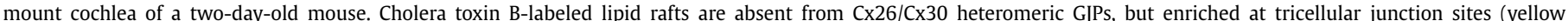

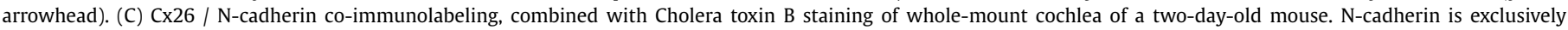

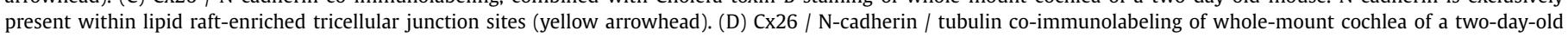

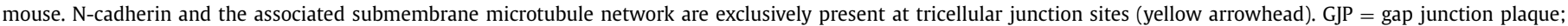

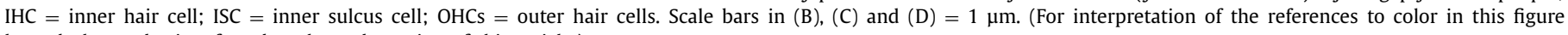
legend, the reader is referred to the web version of this article.)

should represent an advance in understanding the pathogenic significance of these mutations.

The biogenesis of gap junction channels usually relies on preexisting cell-cell junctions, among which are adherens junctions. These are based on cadherin homophilic interactions, which promote the microtubule-mediated trafficking of connexin hemichannels to the cell surface (Shaw et al., 2007). Cadherins are well known as molecular links between two adjacent cells. However, these proteins are also constituents of tricellular junctions (Vanderleest et al., 2018; Yonemura, 2011). These junctions appear to connect three adjacent cells, and they contain a variety of adhesion proteins (Bosveld et al., 2018; Higashi and Miller, 2017). As such, they represent an attractive site for connexin trafficking and gap junction biogenesis in the cochlea.
Here we show that tricellular junctions present at the crossroad between adjacent gap junction plaques (GJPs), provide an unexpected cell surface delivery platform for Cx26/Cx30 oligomers. Using an in situ proximity ligation assay (PLA), we detected the presence of non-junctional Cx26/Cx30 oligomers within lipid raftenriched tricellular junction sites. In addition, we found that the molecular machinery presumably involved in cell surface delivery of Cx26/Cx30 oligomers, including cadherins and associated microtubules, is specifically present within and in the immediate vicinity of tricellular junctions. Using a cadherin neutralizing antibody, we observed that $C \times 26 / C \times 30$ oligomers fail to reach the cell surface and abnormaly accumulate in the cytoplasm. Overall, our results unveil an unexpected role for tricellular junctions in the trafficking and assembly of membrane channels in the cochlea. 


\section{Material and methods}

\subsection{Animals}

Mice of the BALB/C strain were grouped-housed in the animal facility of the University of Liège under standard conditions with food and water ad libitum and were maintained on a 12-h light/dark cycle. All animals were taken care in accordance with the Declaration of Helsinki and following the guidelines of the Belgian ministry of agriculture in agreement with the EC laboratory animal care and use regulation (2010/63/UE, 22 September 2010).

\subsection{Tissue processing and immunostaining}

Cochleae of two-day-old mice were fixed for $2 \mathrm{~h}$ in $4 \%$ formaldehyde. Whole-mount cochleae or organotypic explants were incubated overnight at $4{ }^{\circ} \mathrm{C}$ with primary antibodies directed against connexin 26 (rabbit polyclonal antibody; 1:500; Invitrogen; RRID: AB_2533903), connexin 30 (mouse monoclonal antibody; 1:100; Santa Cruz Biotechnology; sc-514847), N-cadherin (mouse monoclonal antibody, clone GC-4; 1:250; Novus Biologicals; NBP2-21805) and alpha tubulin (rat monoclonal antibody, clone YOL1/34; 1:100; Abcam; RRID: AB_305329). Tissues were la-

\section{A}
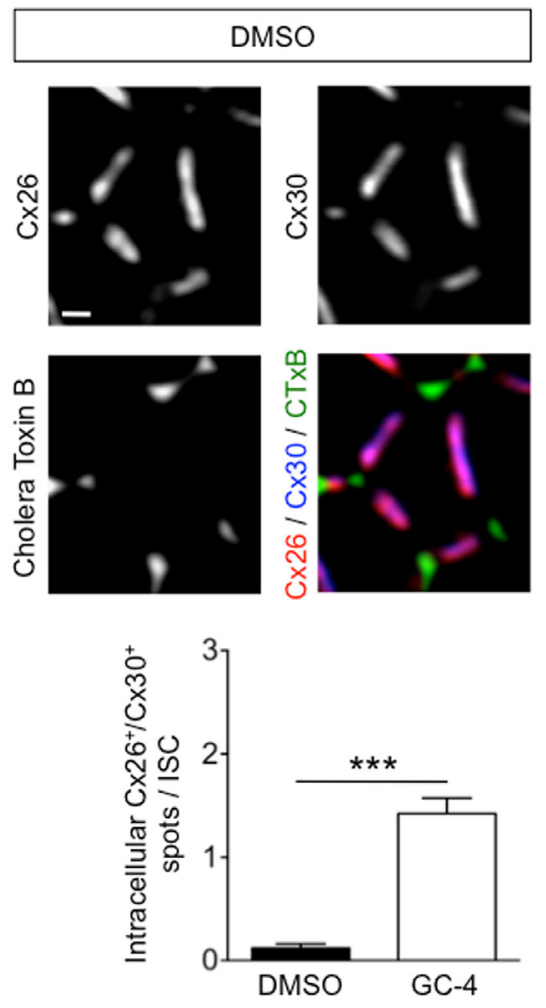
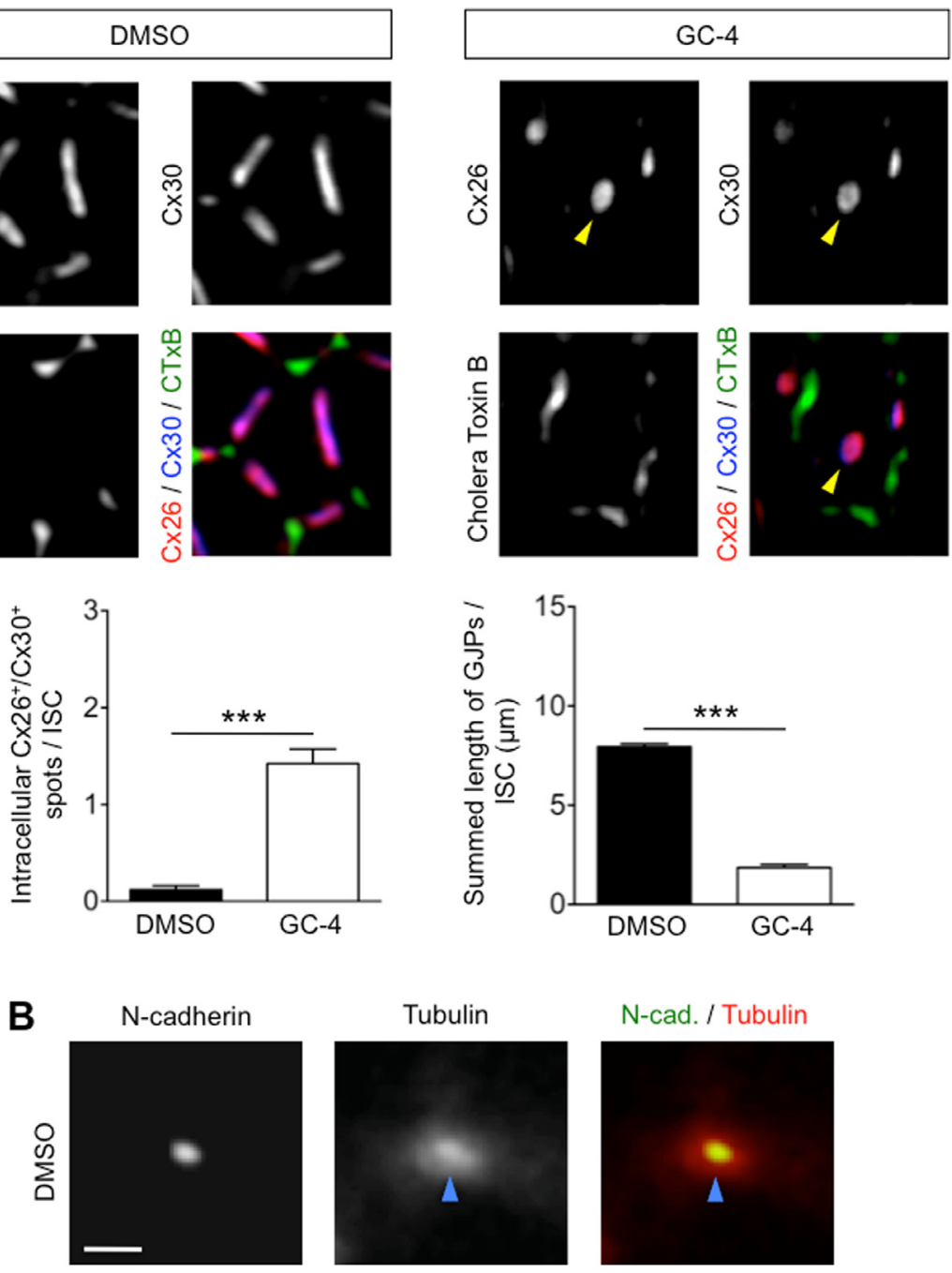

Tubulin
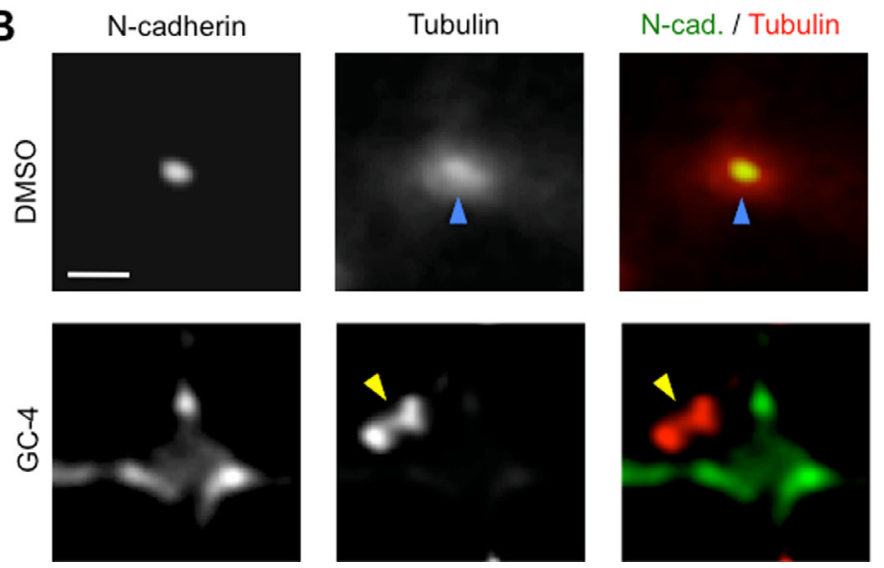

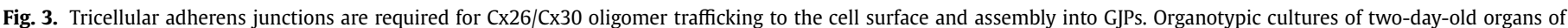

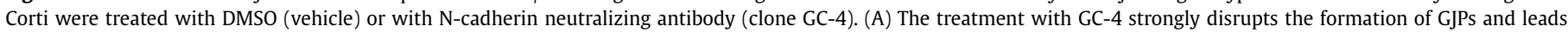

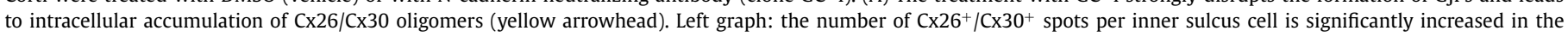

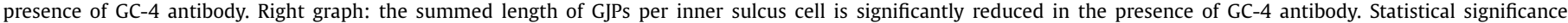

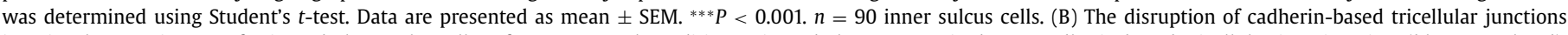

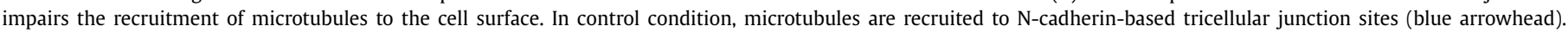

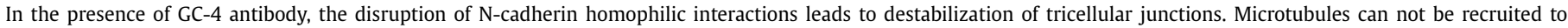

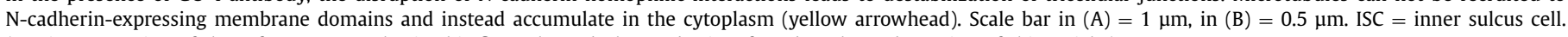
(For interpretation of the references to color in this figure legend, the reader is referred to the web version of this article.) 
A
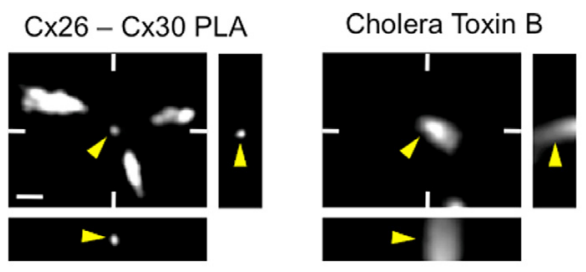

Cx26-Cx30 PLA/

Cholera Toxin B

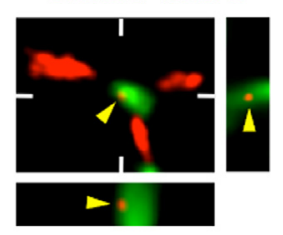

Cx26 ab PLA/

Cholera Toxin B
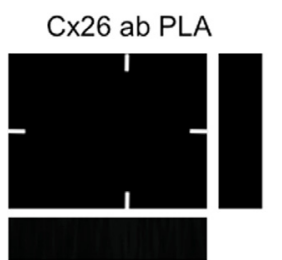

Cholera Toxin B
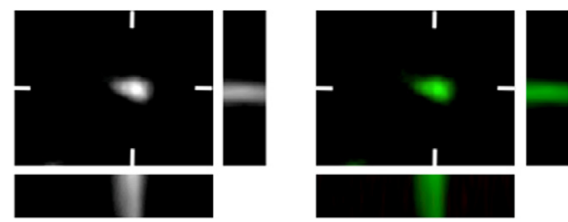

Fig. 2. Non-junctional $C \times 26 / C \times 30$ oligomers are present within lipid raft-enriched tricellular junction sites. (A) In situ proximity ligation assay (PLA) using anti-Cx26 and anti-Cx30 antibodies, combined with Cholera toxin B staining on whole-mount cochlea of a two-day-old mouse. In addition to the GJPs themselves, PLA signals are also detected within lipid raft-enriched tricellular junction sites (yellow arrowhead). (B) The corresponding PLA negative control was performed by omitting the antiCx30 primary antibody. Scale bar in $(A)=1 \mu \mathrm{m}$ in $(A)$ and $(B)$. (For interpretation of the references to color in this figure legend, the reader is referred to the web version of this article.)

beled for lipid rafts using FITC-conjugated Cholera toxin B subunit (1 $\mu \mathrm{g} / \mathrm{mL}$; Sigma-Aldrich; C1655). Tissues were then incubated for $1 \mathrm{~h}$ with either Rhodamine Red X- or FITC- or Cy5-conjugated goat anti-mouse, anti-rabbit or anti-rat IgGs secondary antibodies (Jackson Immunoresearch Laboratories).

\subsection{In situ proximity ligation assay}

In situ PLA (Söderberg et al., 2006) has been previously proven to work on cochlear tissues, and especially with membrane proteins (Defourny et al., 2019a). Whole-mount cochleae were treated and handled as for immunolabeling (see above). Anti-connexin 26 (rabbit polyclonal antibody; 1:500; Invitrogen; RRID: AB_2533903) and anti-connexin 30 (mouse monoclonal antibody; 1:100; Santa Cruz Biotechnology; sc-514847) primary antibodies were incubated with tissues overnight at $4{ }^{\circ} \mathrm{C}$. Oligo-labeled anti-mouse plus and anti-rabbit minus probes (Duolink, Olink Biosciences; DU092101) were then used as recommend by the manufacturer. Negative control was obtained by omitting one of the primary antibody (anticonnexin 30). Tissues were finally labeled for lipid rafts using FITC-conjugated Cholera toxin B subunit $(1 \mu \mathrm{g} / \mathrm{mL}$; Sigma-Aldrich; C1655).

\subsection{In vitro organotypic assay}

Organs of Corti were isolated from two-day-old mice and cultured onto Millicell Culture Insert (Millipore) as previously described (Defourny et al., 2015). Organotypic cultured were incubated for $2 \mathrm{~h}$ with dimethyl sulfoxide (DMSO, Sigma-Aldrich; 276855) or nocodazole (100 $\mu \mathrm{M}$; Sigma-Aldrich; SML1665), and for $6 \mathrm{~h}$ with DMSO or anti-N-cadherin antibody, clone GC-4 (50 $\mu \mathrm{g} / \mathrm{mL}$; Novus Biologicals; NBP2-21805).

\subsection{Confocal microscopy, image analysis and quantifications}

Confocal fluorescence images were acquired using the Olympus Fluoview FV1000 confocal system (Olympus Europa GmbH) with objective LUCPLFLN 40X numerical aperture 0.60, working distance
2.7-4 mm, oil immersion. For comparison between different culture conditions, all preparations were analysed at the same time, using the same acquisition parameters. For each culture condition, intracellular $\mathrm{Cx} 26^{+} / \mathrm{C} \times 30^{+}$spots in 90 inner sulcus cells from three independent experiments were quantified, and data were plotted. For each culture condition, the GJPs of 90 inner sulcus cells from three independent experiments were measured and summed, and data were plotted. Inner sulcus cells were randomly chosen and GJPs were measured using ImageJ software.

\subsection{Statistics}

All data are presented as mean \pm SEM. Data were statistically analysed using two-tailed Student's $t$-test. $P$-values $<0.05$ were considered significant $\left({ }^{* * *} P<0.001\right)$.

\section{Results and discussion}

3.1. $N$-cadherin and associated microtubules are exclusively present at tricellular junction sites between adjacent Cx26/Cx30 gap junction plaques

Gap junction channels are present between the precursor cells of the sensory epithelia in the embryonic cochlea and persist in those cells which differentiate as non-sensory supporting cells. At early postnatal stages, the cells of the nascent inner sulcus display a columnar shape, which progressively evolves to a cuboidal shape with time (Lim and Anniko, 1985). Along the gap junction biogenesis pathway, connexin oligomers are usually delivered from the interior of the cell to non-lipid raft membrane domains. Then, connexin hemichannels diffuse laterally from the peripheral nonjunctional region to assemble into lipid raft GJPs (Hunter et al., 2005; Musil and Goodenough, 1991). The situation is strikingly different concerning the formation of $\mathrm{Cx} 26 / \mathrm{Cx} 30$ heteromeric channels, which connect most non-sensory supporting cell types of the cochlear sensory epithelium, including the inner sulcus cells located medially to the inner sensory hair cell layer (Fig. 1A) (Ahmad et al., 2003; Sun et al., 2005). Cx26/Cx30 GJPs are mostly present basally down the cells, i.e. at some distance away from the apical membrane of these cells (Fig. $1 \mathrm{~A}$ ). We recently observed that Cx26/Cx30 oligomers assemble into non-lipid raft GJPs between adjacent inner sulcus cells, whereas lipid raft microdomains (revealed by Cholera toxin B-subunit labeling) are only found at tricellular junctions, i.e. at the crossroad between three adjacent GJPs (yellow arrowhead in Fig. 1B) (Defourny et al., 2019a). Since lipid rafts are frequently the sites of cell surface delivery of membrane proteins (Bagnat et al., 2000), we wondered whether lipid raftassociated tricellular junctions could promote cell surface delivery of Cx26/Cx30 oligomers. Indeed, tricellular junctions are usually enriched in a variety of adhesion molecules (Bosveld et al., 2018; Higashi and Miller, 2017), among which are cadherin proteins (Vanderleest et al., 2018; Yonemura, 2011), which are known to regulate microtubule dynamics and to promote microtubule anchoring at the cell-cell border (Chausovsky et al., 2000; Shaw et al., 2007; Stehbens et al., 2006). The combined activity of cadherins and microtubules would be consistent with recent findings showing that Cx26/Cx30 GJP formation in the cochlea strongly relies on intact microtubule network (Defourny et al., 2019a). Cadherin proteins are broadly, but differently, expressed in non-sensory supporting cells of the cochlea. Among the latter, inner sulcus cells especially express neural cadherin (N-cadherin) (Etournay et al., 2010), making this protein a candidate for regulating the targeted delivery of $\mathrm{Cx} 26 / \mathrm{Cx} 30$ oligomers to specific membrane domains. At inner sulcus cell-cell borders, we observed that N-cadherin is exclusively present within lipid raft domains, which are confined to 

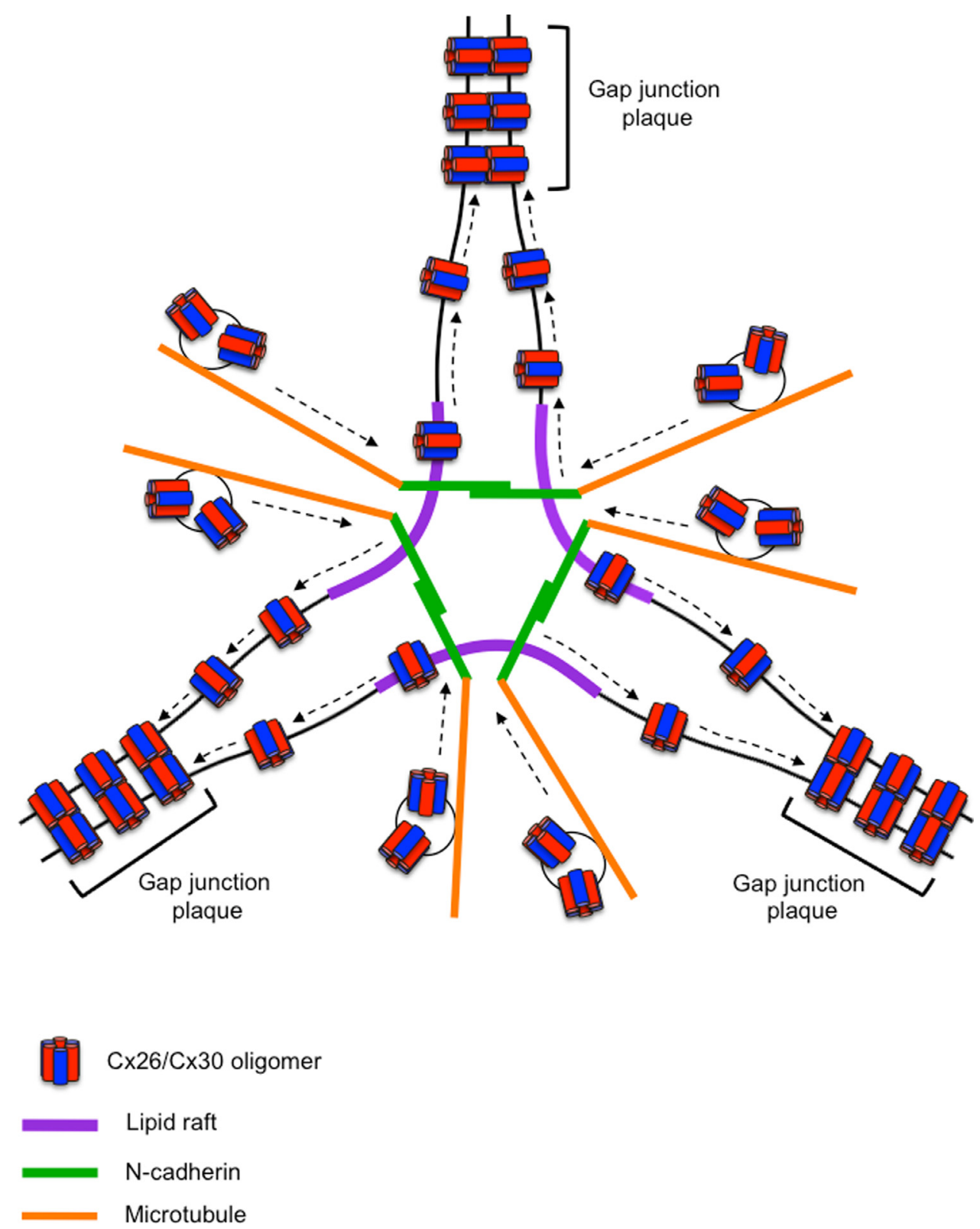

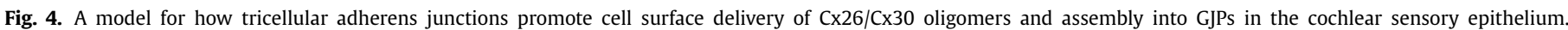

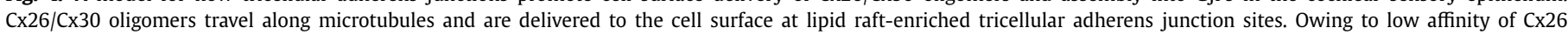

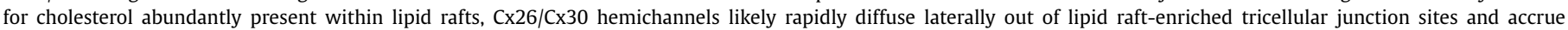
along the outer periphery of a pre-existing GJP, where each hemichannel docks with another from the neighbouring cell to form a new heteromeric channel.

tricellular junction sites (yellow arrowhead in Fig. 1C). This preferential association of $\mathrm{N}$-cadherin with lipid raft fractions is consistent with previous observations (Nakai and Kamiguchi, 2002). In addition, the cadherin-associated submembrane microtubule network, presumably involved in delivering Cx26/Cx30 oligomers to the cell surface, was only observed in the immediate vicinity of $\mathrm{N}$-cadherin-containing tricellular junctions (yellow arrowhead in Fig. 1D). The presence of microtubules at tricellular junction sites was further confirmed using the microtubule-depolymerizing drug nocodazole, which in turn affects the formation of N-cadherinbased adherens junctions (Fig. S1). This finding is consistent with previous observations that dynamic microtubules regulate the local cadherin concentration at cell-cell contacts (Stehbens et al., 2006). Of note is that $\mathrm{Cx} 26 / \mathrm{C} \times 30 \mathrm{GJPs}$ themselves, or their immediate perijunctional regions are devoid of $\mathrm{N}$-cadherin and tubulin, meaning that $\mathrm{Cx} 26 / \mathrm{Cx} 30$ oligomers could not be directly delivered from the interior of the cell to the GJP.

\subsection{Non-junctional $C \times 26 / C \times 30$ oligomers are present within lipid raft-enriched tricellular junction sites}

To detect the presumed transient presence $\mathrm{Cx} 26 / \mathrm{C} \times 30$ oligomers within lipid raft-enriched tricellular junction sites of inner sulcus cells, we used an in situ PLA. Owing to signal amplification, this method should make it possible to reveal the presence of small amounts of proteins that could not be detected using traditional immunofluorescence, such as connexin oligomers in transit on the way to their final destination. Using anti-Cx26 and anti-Cx30 primary antibodies, we detected PLA signals within lipid raft-enriched tricellular junction sites, suggesting the presence of $\mathrm{Cx} 26 / \mathrm{Cx} 30$ oligomers (yellow arrowhead in Fig. 2A). Such PLA signals were observed in $56 \%$ of the Cholera Toxin B-positive sites considered $(n=50)$. To ensure the specificity of PLA signals, an assay was performed by omitting the anti-Cx30 primary antibody. In this case, no PLA signals were observed (Fig. 2B). 
These findings are consistent with previous in vitro data showing the presence of non-junctional Cx26 within lipid rafts, whereas Cx26-containing channels were excluded from lipid raft fractions. On the basis of these results, the authors already suggested that lipid rafts may be involved in trafficking non-junctional membrane Cx26 to non-lipid raft GJPs (Locke et al., 2005). Because of the relatively short half-life of connexins (usually $1-5 \mathrm{~h}$ ), the GJP is in a dynamic state, constantly remodeled through both recruitment of newly synthesized hemichannels to the outer periphery of the GJP and endocytosis of older components from the center of the plaque (Gaietta et al., 2002; Lauf et al., 2002). In agreement with the latter model, we could suggest that, owing to low affinity of Cx26 for cholesterol abundantly present in lipid rafts (Hung and Yarovsky, 2011; Locke and Harris, 2009), Cx26/Cx30 hemichannels rapidly diffuse laterally out of lipid raft-enriched tricellular junctions and accrue along the outer periphery of the pre-existing GJP, where each hemichannel docks with another from the neighbouring cell to form a new heteromeric channel.

\subsection{Tricellular adherens junctions are required for $C \times 26 / C \times 30$} oligomer trafficking and assembly into gap junction plaques

To test the role of cadherin-based tricellular adherens junctions in Cx26/Cx30 oligomer trafficking and assembly into GJPs, we aimed to disrupt $\mathrm{N}$-cadherin homophilic interactions using an anti-N-cadherin neutralizing antibody (clone GC-4) (Puch et al., 2001) in organotypic culture. In this condition, inner sulcus cells display a significant accumulation of $\mathrm{Cx} 26 / \mathrm{C} \times 30$ oligomers in the cytoplasm (yellow arrowhead in Fig. 3A). Moreover, we observed a significant decrease in the summed Cx26/Cx30 GJP length per inner sulcus cell (Fig. 3A). These concomitant defects support the hypothesis that the disruption of $\mathrm{N}$-cadherin-based adherens junctions impairs the trafficking of $\mathrm{Cx} 26 / \mathrm{C} \times 30$ oligomers to the cell surface, which instead accumulate in the cytoplasm. Interestingly, similar defects were previously observed using the microtubuledepolymerizing drug nocodazole (Defourny et al., 2019b), suggesting a cooperation between microtubules and $\mathrm{N}$-cadherin-based adherens junctions in delivering $\mathrm{Cx} 26 / \mathrm{C} \times 30$ oligomers from the interior of the cell to the cell surface. In support of this hypothesis, we found that microtubules can not be anchored at the plasma membrane anymore when $\mathrm{N}$-cadherin homophilic interactions are disrupted with GC-4 neutralizing antibody (yellow arrowhead in Fig. 3B). These observations are consistent with previous data showing that cadherin homophilic interactions recruit microtubules to cell-cell borders (Chausovsky et al., 2000; Shaw et al., 2007; Stehbens et al., 2006). Thus, our results suggest that cadherin-based tricellular adherens junctions promote the microtubule-mediated trafficking of $\mathrm{C} \times 26 / \mathrm{C} \times 30$ oligomers to the cell surface and further assembly into GJPs (Fig. 4). These findings are similar to previous observations showing that $\mathrm{Cx} 40$ and $\mathrm{Cx} 43$ fail to assemble into membrane channels in $\mathrm{N}$-cadherin-depleted cardiac myocytes (Li et al., 2005; Luo and Radice, 2003). As a consequence, the cardiac-specific loss of $\mathrm{N}$-cadherin leads to conduction slowing and arrhythmogenesis (Li et al., 2005).

Over the past decades, several studies have been carried out to decipher the trafficking pathways of $\mathrm{Cx} 26$ and $\mathrm{Cx} 30$. However, a large majority of them reported in vitro data obtained in cell culture with fusion proteins (Cx26-YFP, Cx30-GFP,...) (Kelly et al., 2015; Stout et al., 2015; Thomas et al., 2005). It must be admitted that the behavior of fusion proteins in vitro could be very different to the one of equivalent wild-type proteins in vivo. Nevertheless, our model of Cx26/Cx30 GJP assembly in the cochlea is rather consistent with previous live imaging observations, showing that Cx30-GFP GJPs are replenished at their outer edges (Kelly et al., 2015).

\section{Conclusion}

Until now, tricellular junctions have mainly been considered to provide a mechanical support for epithelial development and morphogenesis (Bosveld et al., 2018; Higashi and Miller, 2017), as well as to maintain epithelial barrier function (Ikenouchi et al., 2005; Nayak et al., 2013). Our data further suggest that they also represent critical organizers of gap junction biogenesis in the cochlea. Indeed, we have shown that tricellular junctions provide an essential cell surface delivery platform for $\mathrm{Cx} 26 / \mathrm{C} \times 30$ oligomers. Overall, our results unveil an unexpected role for tricellular junctions in membrane protein trafficking, and highlight the key dependence of bicellular junctions towards tricellular junctions. However, it has been proposed that active connexin hemichannels in the apical membrane of nascent inner sulcus cells have an important physiological activity during development (Verselis, 2019). In this case, we cannot rule out the possibility that these connexin hemichannels could be delivered to the cell surface by a different way. This question could be the subject of further investigations.

\section{Declaration of Competing Interest}

The authors declare no competing financial interests.

\section{CRediT authorship contribution statement}

Jean Defourny: Conceptualization, Methodology, Formal analysis, Investigation, Writing - original draft, Funding acquisition. Marc Thiry: Writing - review \& editing, Funding acquisition.

\section{Acknowledgments}

We thank the GIGA-Cell Imaging platform.

\section{Funding}

This work was funded by the Belgian Fonds de la Recherche Scientifique-FNRS (F.R.S.-FNRS) (Grant number R.FNRS.4001-6-F) and the Fondation Léon Fredericq (FLF, Faculty of Medicine - University of Liège).

\section{Supplementary materials}

Supplementary material associated with this article can be found, in the online version, at doi:10.1016/j.heares.2020.108137.

\section{References}

Ahmad, S., Chen, S., Sun, J., Lin, X., 2003. Connexins 26 and 30 are co-assembled to form gap junctions in the cochlea of mice. Biochem. Biophys. Res. Commun. 307, 362-368. doi: 10.1016/S0006-291X(03)01166-5

Ambrosi, C., Walker, A.E., Depriest, A.D., Cone, A.C., Lu, C., Badger, J., Skerrett, I.M., Sosinsky, G.E., 2013. Analysis of trafficking, stability and function of human connexin 26 gap junction channels with deafness-causing mutations in the fourth transmembrane helix. PLoS ONE 8, e70916. doi:10.1371/journal.pone.0070916.

Bagnat, M., Keränen, S., Shevchenko, A., Shevchenko, A., Simons, K., 2000. Lipid rafts function in biosynthetic delivery of proteins to the cell surface in yeast. Proc. Natl. Acad. Sci. U.S.A. 97, 3254-3259. doi:10.1073/pnas.060034697.

Beltramello, M., Piazza, V., Bukauskas, F.F., Pozzan, T., Mammano, F., 2005. Impaired permeability to Ins $(1,4,5) \mathrm{P} 3$ in a mutant connexin underlies recessive hereditary deafness. Nat. Cell Biol. 7, 63-69. doi:10.1038/ncb1205.

Berger, A.C., Kelly, J.J., Lajoie, P., Shao, Q., Laird, D., 2014. Mutations in Cx30 that are linked to skin disease and non-syndromic hearing loss exhibit several distinct cellular pathologies. J. Cell Sci. 127, 1751-1764. doi:10.1242/jcs.138230.

Bosveld, F., Wang, Z., Bellaïche, Y., 2018. Tricellular junctions: a hot corner of epithelial biology. Curr. Opin. Cell Biol. 54, 80-88. doi:10.1016/j.ceb.2018.05.002.

Chang, Q., Tang, W., Ahmad, S., Zhou, B., Lin, X., 2008. Gap junction mediated intercellular metabolite transfer in the cochlea is compromised in connexin 30 null mice. PLOS ONE 3, e4088. doi:10.1371/journal.pone.0004088.

Chausovsky, A., Bershadsky, A.D., Borisy, G.G., 2000. Cadherin-mediated regulation of microtubule dynamics. Nat. Cell Biol. 2, 797-804. doi:10.1038/35041037. 
Defourny, J., Mateo Sánchez, S., Schoonaert, L., Robberecht, W., Davy, A., Nguyen, L., Malgrange, B., 2015. Cochlear supporting cell transdifferentiation and integration into hair cell layers by inhibition of ephrin-B2 signalling. Nat. Commun. 6, 7017. doi:10.1038/ncomms8017.

Defourny, J., Peuckert, C., Kullander, K., Malgrange, B., 2019a. EphA4-ADAM10 interplay patterns the cochlear sensory epithelium through local disruption of adherens junctions. iScience 11, 246-257. doi:10.1016/j.isci.2018.12.017.

Defourny, J., Thelen, N., Thiry, M., 2019b. Actin-independent trafficking of cochlear connexin 26 to non-lipid raft gap junction plaques. Hear. Res. 374, 69-75. doi:10.1016/j.heares.2019.01.020.

Defourny, J., Thelen, N., Thiry, M., 2019c. Cochlear connexin 30 homomeric and heteromeric channels exhibit distinct assembly mechanisms. Mech. Dev. 155, 8-14. doi:10.1016/j.mod.2018.10.001.

del Castillo, I., Villamar, M., Moreno-Pelayo, M.A., del Castillo, F.J., Alvarez, A., Telleria, D., Menéndez, I., Moreno, F., 2002. A deletion involving the connexin 30 gene in nonsyndromic hearing impairment. N. Engl. J. Med. 346, 243-249. doi:10.1056/NEJMoa012052.

Etournay, R., Lepelletier, L., Boutet de Monvel, J., Michel, V., Cayet, N., Leibovici, M., Weil, D., Foucher, I., Hardelin, J.P., Petit, C., 2010. Cochlear outer hair cells undergo an apical circumference remodeling constrained by the hair bundle shape. Development 137, 1373-1383. doi:10.1242/dev.045138.

Gaietta, G., Deerinck, T.J., Adams, S.R., Bouwer, J., Tour, O., Laird, D.W., Sosinsky, G.E., Tsien, R.Y., Ellisman, M.H., 2002. Multicolor and electron microscopic imaging of connexin trafficking. Science 296, 503-507. doi:10.1126/science.1068793.

Grifa, A., Wagner, C.A., D’Ambrosio, L., Melchionda, S., Bernardi, F., Lopez-Bigas, N., Rabionet, R., Arbones, M., Monica, M.D., Estivill, X., et al., 1999. Mutations in GJB6 cause nonsyndromic autosomal dominant deafness at DFNA3 locus. Nat. Genet. 23, 16-18. doi:10.1038/12612.

Higashi, T., Miller, A.L., 2017. Tricellular junctions: how to build junctions at the TRICkiest points of epithelial cells. Mol. Biol. Cell 28, 2023-2034. doi:10.1091/ mbc.E16-10-0697.

Hoang Dinh, E., Ahmad, S., Chang, Q., Tang, W., Stong, B., Lin, X., 2009. Diverse deafness mechanisms of connexin mutations revealed by studies using in vitro approaches and mouse models. Brain Res. 1277, 52-69. doi:10.1016/j.brainres. 2009.02.008.

Hung, A., Yarovsky, I., 2011. Gap junction hemichannel interactions with zwitterionic lipid, anionic lipid, and cholesterol: molecular stimulation studies. Biochemistry 50, 1492-1504. doi:10.1021/bi1004156.

Hunter, A.W., Barker, R.J., Zhu, C., Gourdie, R.G., 2005. Zonula occludens-1 alters connexin43 gap junction size and organization by influencing channel accretion. Mol. Biol. Cell 16, 5686-5698. doi:10.1091/mbc.E05-08-0737.

Ikenouchi, J., Furuse, M., Furuse, K., Sasaki, H., Tsukita, S., Tsukita, S., 2005. Tricellulin constitutes a novel barrier at tricellular contacts of epithelial cells. J. Cell Biol. 171, 939-945. doi:10.1083/jcb.200510043.

Kelly, J.J., Shao, Q., Jagger, D.J., Laird, D.W., 2015. Cx30 exhibits unique characteristics including a long half-life when assembled into gap junctions. J. Cell Sci. 128, 3947-3960. doi:10.1242/jcs.174698.

Kelsell, D.P., Dunlop, J., Stevens, H.P., Lench, N.J., Liang, J.N., Parry, G., Mueller, R.F., Leigh, I.M., 1997. Connexin 26 mutations in hereditary non-syndromic sensorineural deafness. Nature 387, 80-83. doi:10.1038/387080a0.

Korver, A.M., Smith, R.J., Paul, D.L., Van Camp, G., Schleiss, M.R., BitnerGlindzicz, M.A., Lustig, L.R., Usami, S.I., Boudewyns, A.N., 2017. Congenital hearing loss. Nat. Rev. Dis. Prim. 3, 16094. doi:10.1038/nrdp.2016.94.

Lauf, U., Giepmans, B.N., Lopez, P., Braconnot, S., Chen, S.C., Falk, M.M., 2002. Dynamic trafficking and delivery of connexons to the plasma membrane and accretion to gap junctions in living cells. Proc. Natl. Acad. Sci. U.S.A. 99, 1044610451. doi:10.1073/pnas.162055899.

Li, J., Patel, V.V., Kostetskii, I., Xiong, Y., Chu, A.F., Jacobson, J.T., Yu, C., Morley, G.E., Molkentin, J.D., Radice, G.L., 2005. Cardiac-specific loss of N-cadherin leads to alteration in connexins with condution slowing and arrhythmogenesis. Circ. Res. 97, 474-481. doi:10.1161/01.RES.0000181132.11393.18.
Lim, D.J., Anniko, M., 1985. Developmental morphology of the mouse inner ear. A scanning electron microscopic observation. Acta Otolaryngol. Suppl. 422, 1-69.

Locke, D., Harris, A.L., 2009. Connexin channels and phospholipids: association and modulation. BMC Biol. 7, 52. doi:10.1186/1741-7007-7-52.

Locke, D., Liu, J., Harris, A.L., 2005. Lipid rafts prepared by different methods contain different connexin channels, but gap junction are not lipid rafts. Biochemistry 44, 13027-13042. doi:10.1021/bi050495a.

Luo, Y., Radice, G.L., 2003. Cadherin-mediated adhesion is essential myofibril continuity across the plasma membrane but not for assembly of the contractile apparatus. J. Cell Sci. 116, 1471-1479. doi:10.1242/jcs.00339.

Musil, L.S., Goodenough, D.A., 1991. Biochemical analysis of connexin43 intracellular transport, phosphorylation, and assembly into gap junctional plaques. J. Cell Biol. 115, 1357-1374. doi:10.1083/jcb.115.5.1357.

Nakai, Y., Kamiguchi, H., 2002. Migration of nerve growth cones requires detergentresistant membranes in a spatially defined and substrate-dependent manner. J. Cell Biol. 159, 1097-1108. doi:10.1083/jcb.200209077.

Nayak, G., Lee, S.I., Yousaf, R., Edelmann, S.E., Trincot, C., Van Itallie, C.M., Sinha, G.P., Rafeeq, M., Jones, S., Belyantseva, I.A., et al., 2013. Tricellulin deficiency affects tight junction architecture and cochlear hair cells. J. Clin. Invest. 123, 40364049. doi:10.1172/JCI69031.

Puch, S., Armeanu, S., Kibler, C., Johnson, K.R., Müller, C.A., Wheelock, M.J., Klein, G. 2001. N-cadherin is developmentally regulated and functionally involved in early hematopoietic cell differentiation. J. Cell Sci. 114, 1567-1577.

Shaw, R.M., Fay, A.J., Puthenveedu, M.A., von Zastrow, M., Jan, Y.N., Jan, L.Y., 2007. Microtubule plus-end-tracking proteins target gap junctions directly from the cell interior to adherens junctions. Cell 128, 547-560. doi:10.1016/j.cell.2006.12. 037.

Söderberg, O., Gullberg, M., Jarvius, M., Ridderstrale, K., Leuchowius, K.J., Jarvius, J. Wester, K., Hydbring, P., Bahram, F., Larsson, L.G., et al., 2006. Direct observation of individual endogenous protein complexes in situ by proximity ligation. Nat. Methods 3, 995-1000. doi:10.1038/nmeth947.

Stehbens, S.J., Paterson, A.D., Crampton, M.S., Shewan, A.M., Ferguson, C., Akhmanova, A., Parton, R.G., Yap, A.S., 2006. Dynamic microtubules regulate the local concentration of E-cadherin at cell-cell contacts. J. Cell Sci. 119, 1801-1811. doi:10.1242/jcs.02903.

Stout Jr., R.F., Snapp, E.L., Spray, D.C., 2015. Connexin type and fluorescent proteinfusion tag determine structural stability of gap junction plaques. J. Biol. Chem. 290, 23497-23514. doi:10.1074/jbc.M115.659979.

Sun, J., Ahmad, S., Chen, S., Tang, W., Zhang, Y., Chen, P., Lin, X., 2005. Cochlear gap junctions coassembled from $\mathrm{Cx} 26$ and 30 show faster intercellular $\mathrm{Ca} 2+$ signaling than homomeic counterparts. Am. J. Phys. Cell Phys. 288, C613-C623. doi:10.1152/ajpcell.00341.2004.

Thomas, T., Jordan, K., Simek, J., Shao, Q., Jedeszko, C., Walton, P., Laird, D.W., 2005. Mechanisms of Cx43 and Cx26 transport to the plasma membrane and gap junction regeneration. J. Cell Sci. 118, 4451-4462. doi:10.1242/jcs.02569.

Vanderleest, T.E., Smits, C.M., Xie, Y., Jewett, C.E., Blankenship, J.T., Loerke, D., 2018. Vertex sliding drives intercalation by radial coupling of adhesion and actomyosin networks during Drosophila germband extension. Elife 7, e34586. doi:10.7554/eLife.34586.

Verselis, V.K., 2019. Connexin hemichannels and cochlear function. Neurosci. Lett. 695, 40-45. doi:10.1016/j.neulet.2017.09.020.

$\mathrm{Xu}, \mathrm{J}$., Nicholson, B.J., 2013. The role of connexins in ear and skin physiology - functional insights from disease-associated mutations. Biochim. Biophys. Acta 1828 167-178. doi:10.1016/j.bbamem.2012.06.024.

Yonemura, S., 2011. Cadherin-actin interactions at adherens junctions. Curr. Opin. Cell Biol. 23, 515-522. doi:10.1016/j.ceb.2011.07.001.

Zdebik, A.A., Wangemann, P., Jentsch, T.J., 2009. Potassium ion movement in the inner ear: insights from genetic disease and mouse models. Physiol. Bethesda 24, 307-316. doi:10.1152/physiol.00018.2009. 